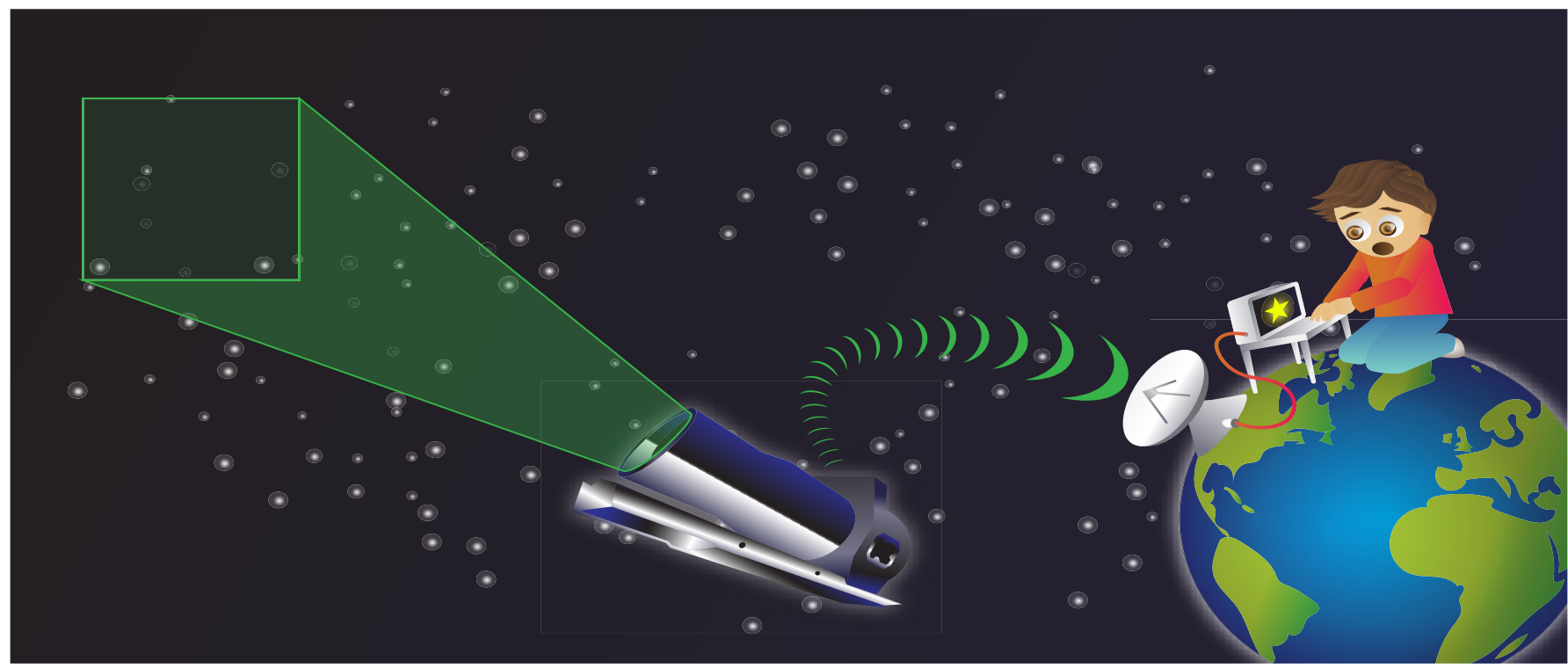

\title{
WHAT DO "YELLOWBALLS" HAVE TO DO WITH THE BIRTH OF NEW STARS?
}

\section{Grace Wolf-Chase', Charles Kerton ${ }^{2}$}

${ }^{1}$ The Adler Planetarium, Chicago, IL, USA, ${ }^{2}$ Department of Physics and Astronomy, lowa State University, Ames, IA, USA

\section{REVIEWED BY: \\ FRIDA \\ 16 YEARS OLD \\ TAYLOR \\ 16 YEARS OLD}

Where do stars come from? Human beings have thought about this question for thousands of years and have proposed many different explanations, but scientists have only had the technology to observe the places where stars are forming for a few decades. This is because stars form inside cold "dusty" clouds in space that are invisible to our eyes and invisible to telescopes that study visible light. Fortunately, we have many instruments today that can record light that our eyes cannot see, and we can use familiar colors to represent this light. Even very cold objects give off infrared light, so we can use this type of light to explore how the dusty clouds produce stars. People from around the world have helped scientists identify an early stage in the development of stars, called "yellowballs," by searching infrared images in an important science project called the Milky Way Project.

\section{SCIENCE FOR EVERYONE}

Modern science generates lots of data, and sometimes the amount of data generated by a scientific project is simply too much for a single scientist, or even a small group of scientists, to handle in a reasonable amount of 
INTERSTELLAR

\section{MEDIUM}

Diffuse dust and gas that is found between the stars in a galaxy. The coldest and densest regions of the interstellar medium are where stars can form. These regions are opaque to visible light, thus appearing as dark patches in optical images of our Galaxy.

\section{FIGURE}

The Milky Way. Astronomer José Francisco Salgado took this photo of the center of our Galaxy in Cedar Flat, Calif., on May 20, 2010.

Below it stands the

Combined Array for Research in Millimeterwave Astronomy instrument made up of 23 radio telescopes.

Credit: Adler Planetarium, Chicago, IL, USA and José Francisco Salgado. time. In these cases, professional scientists can sometimes call on the help of enthusiastic "citizen scientists." A citizen scientist is anyone who works on a real science project, designed by a team of professional scientists who need many human eyes (or in some cases, ears) in order to accomplish the goals of the project. The Zooniverse website [1] currently features more than three-dozen such science projects involving over 1.3 million citizen scientists, including the Milky Way Project, where citizen scientists help study star formation in our home galaxy.

\section{OUR HOME GALAXY}

Our Sun belongs to a very large group of stars that we call the Milky Way Galaxy. The Milky Way Galaxy is pretty flat, like a huge disk. If you have ever stargazed from a very dark place, you may have noticed a bright band of light stretching across the sky (see Figure 1). This band of light is made up of numerous stars that lie in the disk of the Milky Way. Most of these stars are far too faint for human eyes to see individually. From the darkest locations on Earth, you may be able to see a few thousand stars, from a small town, perhaps a few hundred, and from a big city, probably no more than a few dozen. In reality, there are a few hundred billion stars in our Galaxy (one hundred billion $=100,000,000,000$ ), but you would not be able to see the light from more than a small fraction of these stars even if you used the most powerful telescope! This is because the interstellar medium, or the space between the stars, contains enormous clouds of gas and dust, called nebulae. The coldest and densest of these nebulae block the light from stars within and behind these clouds.

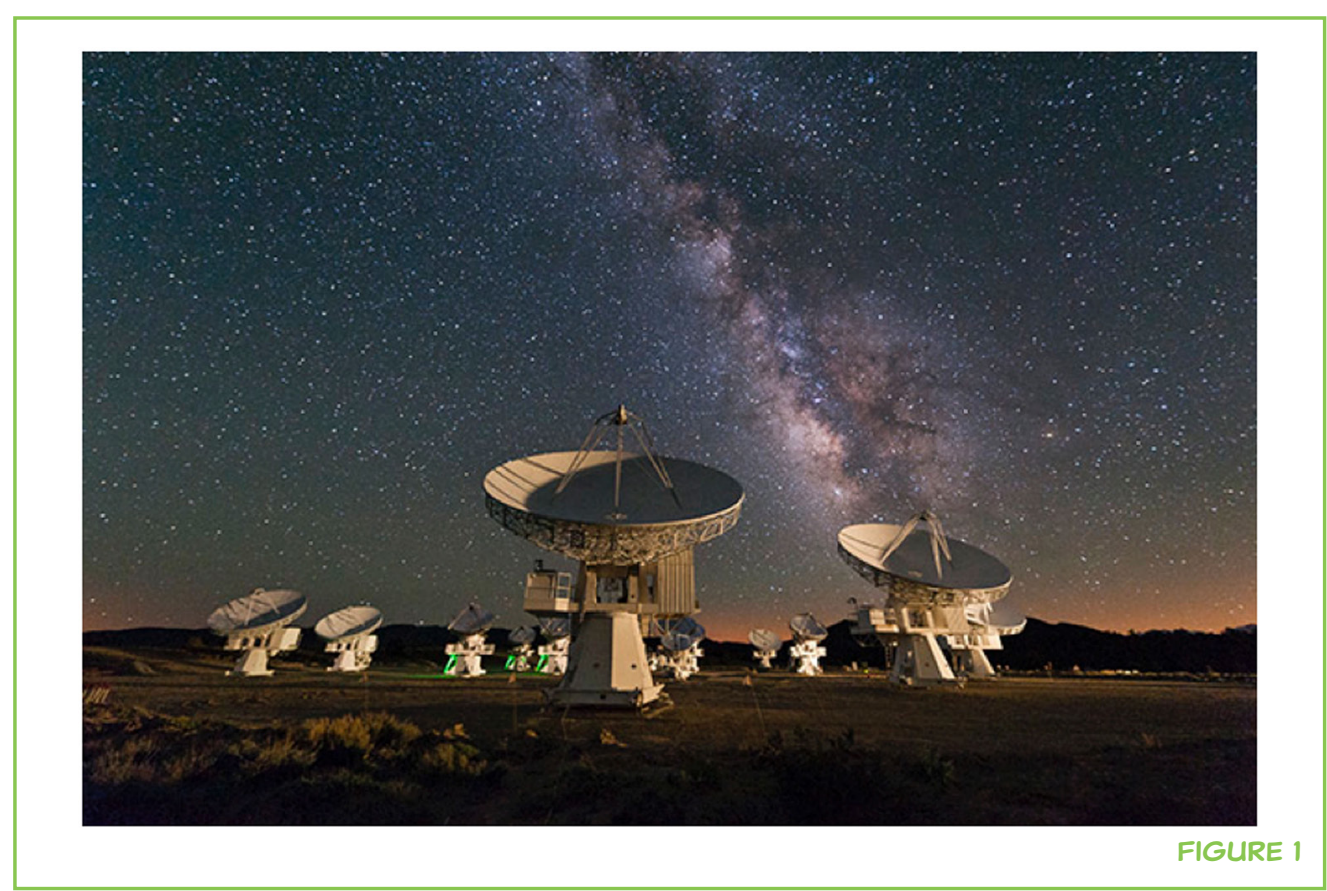




\section{INFRARED LIGHT}

A type of light that has a longer wavelength than visible light. The longer wavelength of infrared light means it can pass through dusty regions of the interstellar medium more easily than visible light.

\section{SPITZER SPACE TELESCOPE}

A smaller cousin of the Hubble Space Telescope. Spitzer consists of a $0.85-m$ diameter telescope plus instrumentation designed to observe infrared light. It was launched by NASA in 2003 into an Earth-trailing orbit around the Sun.

\section{FIGURE 2}

An Infrared View of the Milky Way.

This image of a portion of the Milky Way toward the center of our Galaxy uses blue, green, and red to represent different colors of infrared light captured by the Spitzer Space Telescope. Other colors are formed where any of these three colors overlap (see the inset).

Credit: NASAJJPL-Caltech/ Univ. of Wisconsin.

\section{THE HIDDEN BIRTHPLACES OF STARS}

Stars form in the densest and dustiest regions of the interstellar medium, where it is impossible to study the stars using a regular telescope. Fortunately, over the past few decades, astronomers have developed telescopes and detectors that are sensitive to invisible infrared light, which is a type of light that can pass through the dusty regions of the interstellar medium. These instruments allow them to detect cool objects and to peer inside nebulae to study how stars form. Initially a newly formed star is too cold to shine in visible light, but it does shine brightly in infrared light. As the star forms, it heats up and starts to shine more and more brightly in visible light. Unfortunately, this visible light from the star gets absorbed by the dust in the surrounding nebula and never makes it out of the cloud. However, infrared light from the star can make it through the cloud, plus the surrounding dust is heated by the starlight and also becomes very bright in infrared light. Astronomers thus can use infrared light to study both the forming star and its surroundings [2].

Figure 2 shows an infrared image of a portion of our Galaxy. As you look at it, you may be wondering how we make a picture of light we cannot see with our eyes. The "trick" is to use a powerful computer program to turn infrared colors we cannot see into colors of visible light (often blue, green, and red). Different colors of light bring us information about the composition of different types of objects (what they are made of) and physical conditions (for example, temperature). In the infrared image shown in Figure 2, every blue point is a star, while certain types of gas molecules and dust that make up nebulae show up in green and red. When two or more of these colors overlap in the image, you get a different color. For example, green + red make yellow (see the inset on Figure 2).

The Milky Way Project features infrared images that were taken by the Spitzer Space Telescope. This project was created because scientists need help to catalog and study the information revealed in the hundreds of thousands of infrared

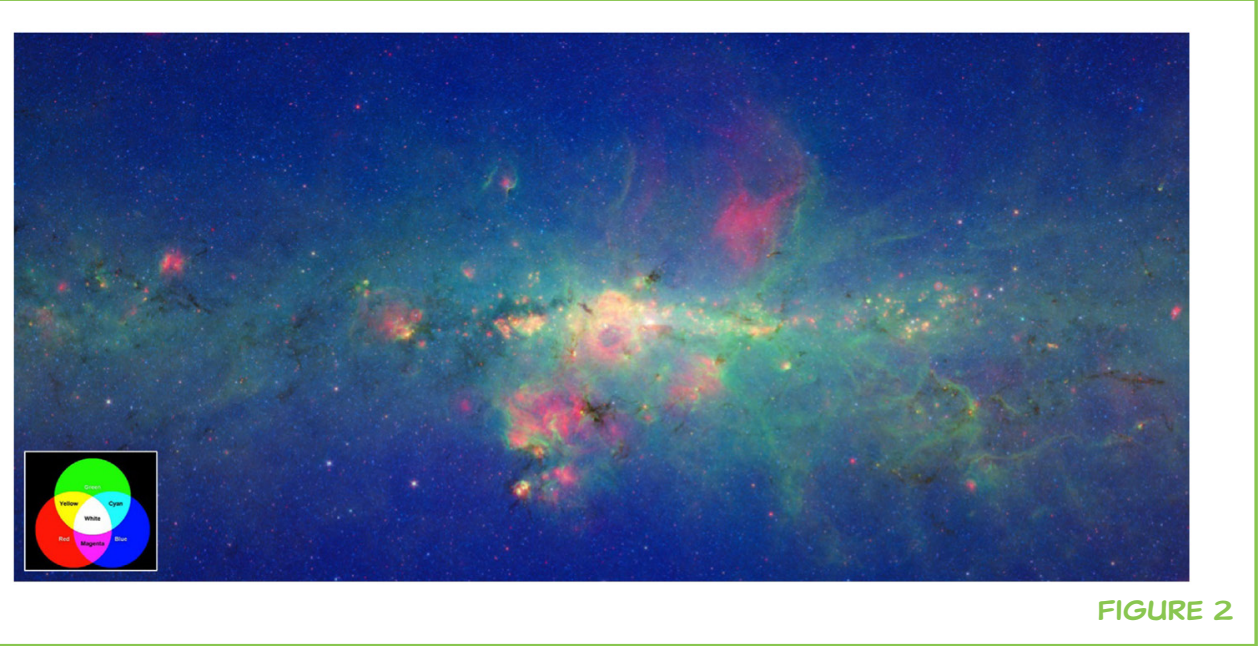




\section{SOLAR WIND}

The highly magnetized flow of charged particles, primarily protons and electrons, streaming from the Sun. The solar wind moves at velocities of several hundred kilometers/second.

\section{FIGURE 3}

The Evolution of a Bubble.

This is a sequence of images that illustrates stages in the development of a bubble around massive young stars. The "yellowball" stage, identified by citizen scientists, is shown in the middle panel.

Credit: NASAJJPL-Caltech. images of the Milky Way that were provided by the Spitzer Space Telescope. Citizen scientists are inspecting images like the ones that were combined to produce Figure 2 in order to help professional scientists learn how stars form.

\section{A SURPRISING DISCOVERY..}

Some of the features that we asked people to help us find in these images look like red circles (or ovals) with green rims, like the one you can see in the right panel of Figure 3 [3]. The red color primarily represents very small dust particles and the green color primarily represents warm gas molecules that are made up of many hydrogen and carbon atoms. Just as a ball would look flat in a 2-dimensional picture, these red and green features look like circles in the images that the citizen scientists examine, but in space these circles are actually more like "bubbles," since space is 3-dimensional.

Massive young stars (where "massive" is anything more than about 10 times the mass of our Sun) produce these bubbles as radiation and "winds" from the stars push against the surrounding nebula. "Winds" from a star are not really wind like we experience here on earth, but are streams of tiny charged particles like the protons and electrons that make up the solar wind. The young stars heat the gas inside a bubble to very high temperatures, so high that the molecules are destroyed, although some dust particles survive. This is why, in the images, the bubble interiors are red and the rims, where the molecules survive, are green.

While Milky Way Project citizen scientists were inspecting images for bubbles, they noticed small, roundish yellow objects in many of the images and began to wonder what these were. We asked the citizen scientists to continue to mark

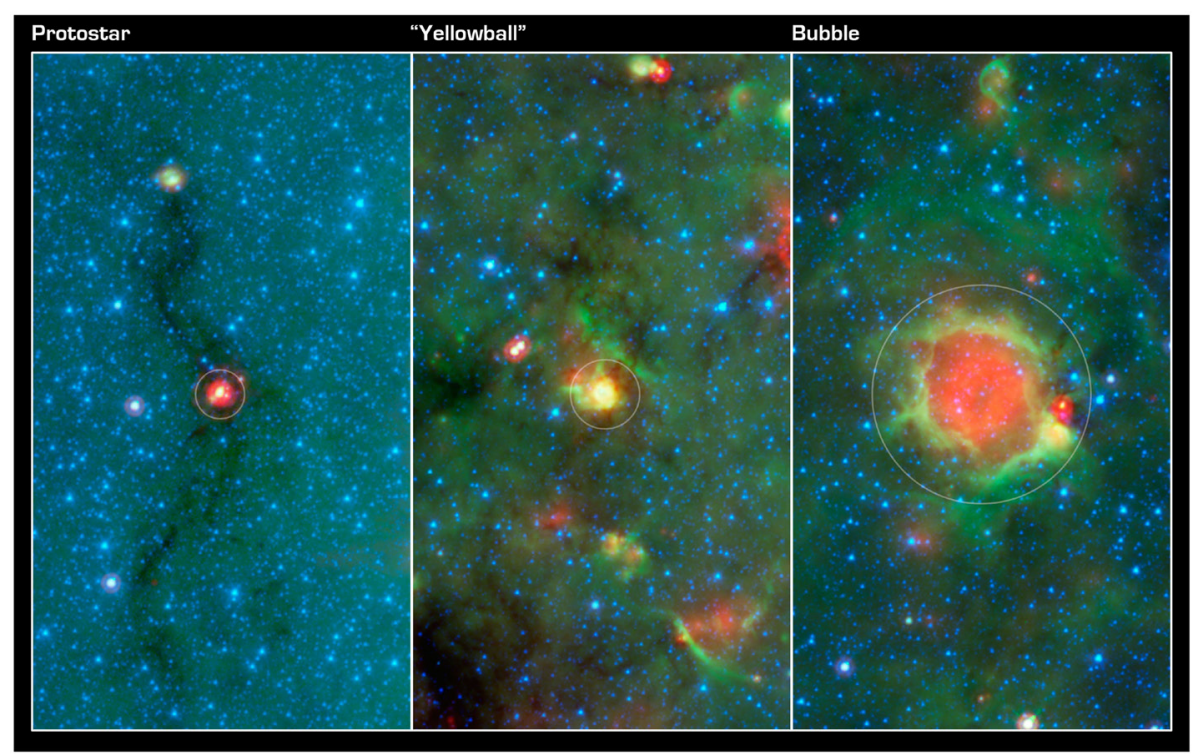

FIGURE 3 
PROTOSTAR

A large, spherical concentration of gas and dust that is still in the process of contracting to form a star. The core of a protostar is not hot and dense enough to be powered by fusion reactions. Once it is, fusion starts and the protostar becomes a star.

these objects in the images so we could study them further. Since the color yellow appears where red and green overlap in these images, we hypothesized that the "yellowballs," as we call them, might be very young versions of the larger bubbles, where most of the gas molecules near the young stars have not been destroyed yet [4].

The first two panels of Figure 3 show early stages in the evolution of a bubble. The long dark feature in the left panel is a very cold nebula, too cold to be detected by the infrared telescope. In some of these very cold nebulae, gravity is strong enough to pull the cold gas and dust together to form new stars. The red "dot" you can see in the dark nebula shows where protostars are starting to warm up the dust. A protostar is like an infant star - it is still growing as the surrounding matter is pulled together by gravity. This "infant" stage lasts about 10,000 years. Once protostars get hot enough, the matter surrounding them begins to expand and both dust and gas molecules glow in infrared light, creating a yellowball (center panel) that is about 100,000 years old. Most yellowballs are larger than our Solar System, but smaller than the distance between our Sun and the next closest star, Proxima Centauri, and yet each yellowball may be producing dozens or even hundreds of stars.

Over 1,000,000 years or so, the yellowball transforms into the mature bubble shown in the right panel of Figure 3. After a few million years the bubble itself will disperse into the general interstellar medium, leaving behind the newly formed stars that will, in turn, become part of the disk of the Milky Way Galaxy.

\section{HUNDREDS OF STARS}

An example of what a bubble may look like after a few million years is given by the Orion nebula. This favorite of both professional and amateur astronomers is hot enough to glow in visible light, and is the closest example of massive star formation in the Galaxy. Perhaps you have even seen this nebula through a small telescope or pair of binoculars during an evening of stargazing? While the nebula is powered by only a handful of massive stars, many hundreds of smaller stars like the Sun, complete with cold disks of material where planets are probably forming, are also found in the nebula along with the more massive stars. Figure 4 shows a view of the Orion nebula that was taken by the Hubble Space Telescope [5]. It includes zoomed-in images of planetary systems that are currently forming in the nebula.

There are good reasons to think that our Solar System formed in an environment similar to the Orion nebula about five billion years ago, so learning about how bubbles evolve will help us understand what conditions may have been like when the Earth formed. 


\section{FIGURE 4}

Planetary Systems Now Forming in Orion.

This is a visible light image of the Orion nebula, put together by the Hubble Space Telescope. Each of the zoomed-in images shows a planetary system forming in the nebula. The top zoomed-in image is roughly the size of our Solar System (about six billion miles across). The entire nebula is several light-years across

(1 light-year is about six trillion miles:

$6,000,000,000,000)$ and about 1,500 light-years away from the Sun.

Credit: NASA, ESA, M. Robberto (STScl/ESA), the HST Orion Treasury Project Team, \& L. Ricci (ESO).

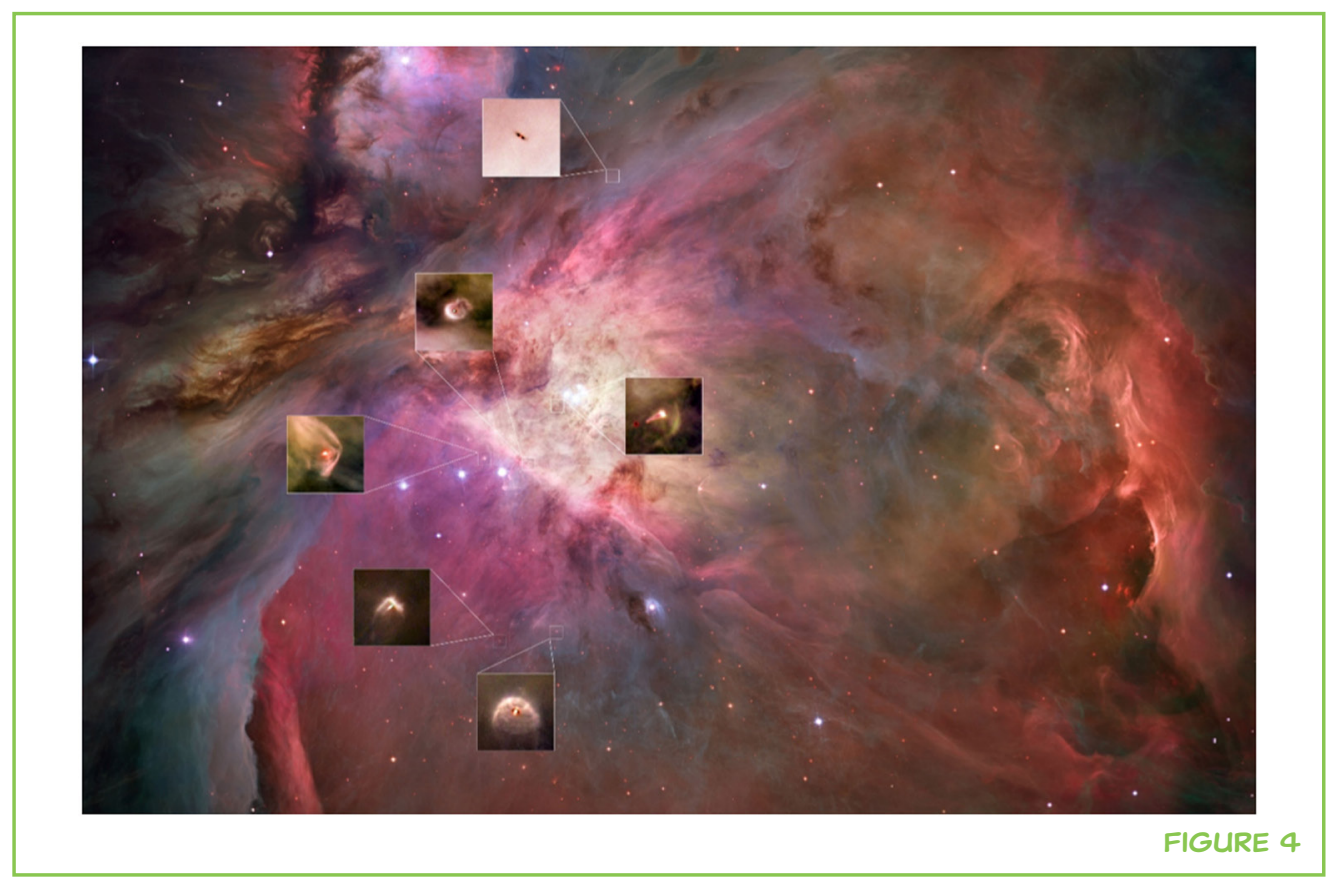

\section{"EUREKA!" OR "THAT'S FUNNY"?}

The key to identifying the earliest stages in the development of star clusters was finding the best combination of infrared colors to highlight important features. Using a different combination of infrared colors, the yellowballs would not pop out. Ideally, astronomers want to look at as many different colors of light as they can, but the reality is they can not build instruments that do everything. Before building astronomical instruments, scientists need to think about what types of observations will yield the most information about the objects they are trying to understand. The infrared colors in the Spitzer Space Telescope images were chosen to examine features like bubbles, but we did not know how well these colors would highlight the younger yellowball stage!

Isaac Asimov was a famous twentieth century science fiction writer who once said, "The most exciting phrase to hear in science, the one that heralds new discoveries, is not 'Eureka!' but 'That's funny." Now, thanks to the curiosity of our citizen scientists, including the first person who thought "That's funny" and shared that thought about the yellowball with others, we have a catalog of about 1,000 of these objects. Using this new catalog, astronomers can design future observations that will tell them more about how yellowballs evolve into stellar nurseries that may be giving birth to other planets like our Earth.

\section{REFERENCES}

1. Zooniverse. 2015. Available at: www.zooniverse.org

2. Cool Cosmos. 2015. Available at: http://coolcosmos.ipac.caltech.edu/ 
3. Simpson, R. J., Povich, M. S., Kendrew, S., Lintott, C. J., Bressert, E., Arvidsson, K., et al. 2012. The milky way project first data release: a bubblier galactic disk. Mon. Not. R. Astron. Soc. 424:2442-60. doi:10.1111/j.1365-2966.2012.20770.x.

4. Kerton, C. R., Wolf-Chase, G., Arvidsson, K., Lintott, C. J., and Simpson, R. J. 2015. The milky way project: what are yellowballs? Astrophys. J. 799:153-61. doi:10.1088/0004-637X/799/2/153.

5. O'Dell, C. R., Wen, Z., and Hu, X. 1993. Discovery of new objects in the Orion nebula on HST images - shocks, compact sources, and protoplanetary disks. Astrophys. J. 410:696-700. doi:10.1086/172786.

SUBMITTED: 29 June 2015; ACCEPTED: 26 Oct 2015; PUBLISHED ONLINE: 16 November 2015.

EDITED BY: Shane L Larson, Northwestern University, USA.

CITATION: Wolf-Chase G and Kerton C (2015) What do "yellowballs" have to do with the birth of new stars? Front. Young Minds 3:16. doi:10.3389/frym.2015.00016

COPYRIGHT @) 2015 Wolf-Chase and Kerton. This is an open-access article distributed under the terms of the Creative Commons Attribution License (CC BY). The use, distribution or reproduction in other forums is permitted, provided the original author(s) or licensor are credited and that the original publication in this journal is cited, in accordance with accepted academic practice. No use, distribution or reproduction is permitted which does not comply with these terms.

\section{REVIEWED BY:}

\section{FRIDA, 16 YEARS OLD}

Hi! I am Frida and I am an artist and an intern at the Adler Planetarium in Chicago.

\section{TAYLOR, 16 YEARS OLD}

I'm Taylor and I'm a citizen science intern at the Adler Planetarium. I'm going to be a junior at Lane Tech College Prep.

\section{AUTHORS}

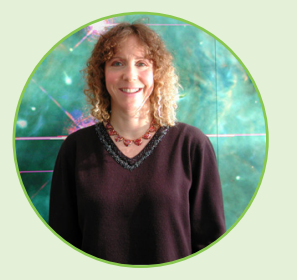

\section{GRACE WOLF-CHASE}

My two greatest interests as a child were astronomy and competitive figure skating. Visiting museums played a huge part in stirring my imagination and inspiring my thoughts about space, so I love being an astronomer at the Adler Planetarium. I am fascinated by questions relating to how stars and planets form, and what they can tell us about how our own world came to be. Being able to share my excitement for science with others, including my three children, has been one of the best parts of my job. Although I do not skate as much as I used to, I do enjoy swimming or working out every morning. 


\section{CHARLES KERTON}

I am a teacher and researcher in the Department of Physics \& Astronomy at lowa State University. My love of astronomy started with viewing Saturn, the Moon, and Orion through a small 60-mm diameter "department store" telescope at an early age, and I have not stopped enjoying astronomy since. Away from the telescope and computer I enjoy gardening and hiking (in the summer), ice skating (in the winter), and reading sci-fi and history (all year). 